\title{
Radiological Diagnosis of Soft Tissue Tumors in Adults: MRI Imaging of Selected Entities Delineating Benign and Malignant Tumors
}

\author{
Radiologische Diagnostik von Weichteiltumoren im \\ Erwachsenenalter: MRT-Bildgebung ausgewählter Entitäten \\ mit Abgrenzung zwischen benignen und malignen Tumoren
}

\author{
Authors \\ Catharina Silvia Lisson, Christoph Gerhard Lisson, Meinrad Beer, Stefan Andreas Schmidt
}

\begin{abstract}
Affiliation
Department of Diagnostic and Interventional Radiology, University-Hospital of Ulm, Germany

Key words

muscular, skeletal-appendicular, skeletal-axial, soft tissues, neoplasms
\end{abstract}

received 19.02.2018

accepted 11.08.2018

\author{
Bibliography \\ DOI https://doi.org/10.1055/a-0715-2205 \\ Published online: 18.12 .2018 \\ Fortschr Röntgenstr 2019; 191: 323-332 \\ (c) Georg Thieme Verlag KG, Stuttgart · New York \\ ISSN 1438-9029
}

\section{Correspondence}

Priv.-Doz. Dr. Stefan Andreas Schmidt

Diagnostic and Interventional Radiology, Universitätsklinikum Ulm Klinik für Diagnostische und Interventionelle Radiologie, Albert-Einstein-Allee 23, 89081 Ulm, Germany

Tel.: ++ 49/7 31/50061003

Fax: $++49 / 731 / 50061005$

stefan.schmidt@uniklinik-ulm.de

\section{ABSTRACT}

Objective MRI is the most important and sensitive imaging modality in the differentiation of unclear soft tissue tumors. A systematic approach helps to narrow down the large number of possible differential diagnoses.

Method Our review systematically compares MRI characteristics of the major soft-tissue masses and aims to gain access to these often difficult tumor entities.
Results and conclusion MRI, as the most important modality in the imaging of soft tissue tumors, allows a more detailed classification of the tumor entity and in many cases a differentiation between benign and malignant masses.

Key points:

- MRI is the method of choice for classifying unclear soft tissue tumors.

- A systematic approach may differentiate benign from unclear lesions.

- In cases of doubt, a biopsy should be performed to rule out malignancy.

\section{Citation Format}

- Lisson CS, Lisson CG, Beer M et al. Radiological Diagnosis of Soft Tissue Tumors in Adults: MRI Imaging of Selected Entities Delineating Benign and Malignant Tumors. Fortschr Röntgenstr 2019; 191: 323-332

\section{ZUSAMMENFASSUNG}

Ziel In der Differenzierung unklarer Weichteiltumoren stellt die MRT die wichtigste und sensitivste Bildgebungsmodalität dar. Dabei hilft eine systematische Herangehensweise, die große Zahl möglicher Differenzialdiagnosen einzuengen.

Methode Unsere Übersichtsarbeit stellt systematisch die MR-tomografischen Charakteristika der wichtigsten weichteiligen Raumforderungen gegenüber und will helfen, einen Zugang zu diesen oft schwierigen Tumorentitäten zu erhalten. Ergebnisse und Schlussfolgerung Durch die MRT als wichtigster Baustein in der Bildgebung von Weichteiltumoren ist eine nähere Einordnung der Tumorentität und oft auch eine Differenzierung zwischen benignen und malignen Raumforderungen möglich.

\section{Introduction}

On the whole, malignant soft tissue tumors account for about $1 \%$ of malignant tumors in adults; among children they make up about $15 \%$ of malignancies. Overall, the ratio of benign to malig- nant tumors is given as 100:1 [1]. Nevertheless, in the case of a clinically identified new soft tissue tumor, further diagnosis and therapy should be based on a standardized procedure in order to take advantage of prompt initiation of therapy even with less common malignancies ( $\$$ Fig. 1 based on [2]). MRI plays an out- 
Selection of adjuvant therapy and follow-up in Tumor Board

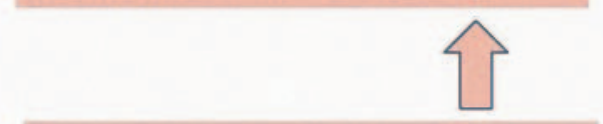

Extensive resection, neoadjuvant therapy as needed

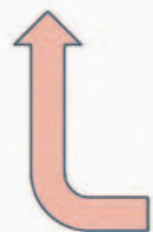

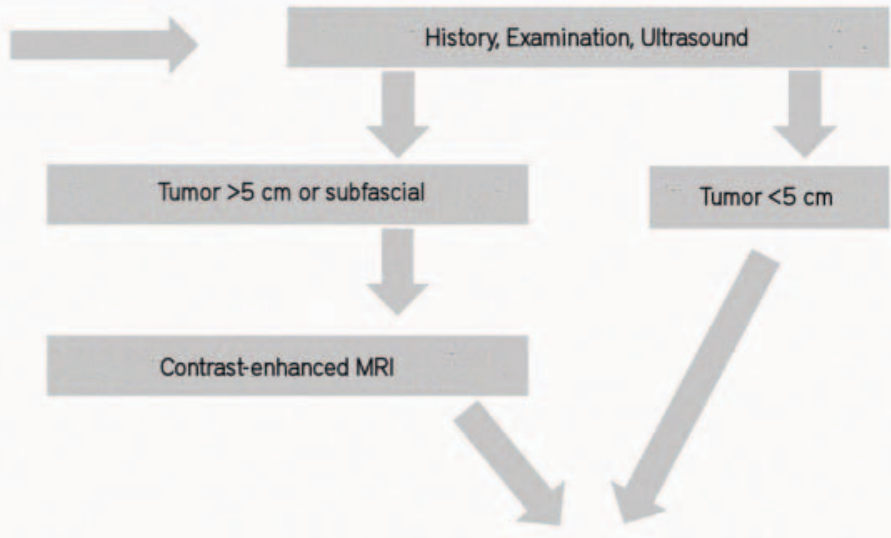

Biopsy and histopathological finding

Soft tissue carcinoma:

$\rightarrow$ Staging (Thoracic CT, PET CT as needed

$\rightarrow$ Interdisciplinary Tumor Board

- Fig. 1 Flow chart of diagnosis and therapy of unclear soft tissue tumors, based on [2].

standing role in diagnosis, since the large initially large number of differential diagnoses can be narrowed further due to specific differences in the signal behavior of the tumor in the various MR sequences. In many cases a definitive diagnosis is possible which can spare the patient an invasive investigation by biopsy or even surgery. This review focuses on the image-based description of selected tumor entities and their systematic characterization.

\section{Common soft tissue tumors and their characteristics in imaging}

In an MRI some soft tissue tumors exhibit signal characteristics which permit a distinction of possible differential diagnoses, thus aiding the radiologist and, in the best case, even avoiding a biopsy. The following examples describe masses which are common, but which also have a typical appearance in imaging on the other hand.

\section{Adipocyte tumors}

Lipomas are the most common soft tissue tumors with an incidence of 2.1 per 100 cases. In conventional X-rays or CT, lipomas are radiolucent isointense to subcutaneous adipose tissue in all MRI sequences [3] ( $\triangleright$ Fig. 2).

A classical lipoma consists solely of adipose tissue without nodular components or septations [4]. However, a significant portion of benign lipomas also contain non-fatty components. In a study by Kransdorf et al., [4] 31 \% of lipomas exhibited non-fatty elements in the form of fatty necroses with calcifications, fibroses

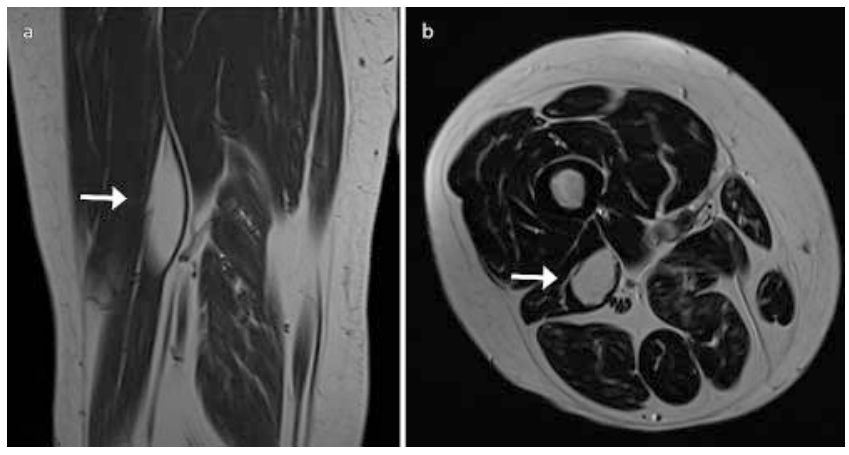

- Fig. 2 56-tear-old male with posterior intramuscular lipoma on thigh. a In coronal T1 TSE a hyperintense lesion, isointense to subcutaneous adipose tissue (arrow). $\mathbf{b}$ In an axial T2 TSE the smoothmargined mass is likewise homogeneously hyperintense without evidence of thickened septa or nodular components (arrow).

and other changes. It is also worth mentioning that although subforms such as angiolipoma or myolipoma also consist for the most part of fatty parts, here the non-fatty components may make it difficult to diagnose a benign lipoma [5, 6]. The important differential diagnosis of a lipoma is the well-differentiated liposarcoma, which also consists largely of fatty parts. In contrast, other subtypes such as the myxoid liposarcoma have almost no visible patty parts [7]. Characteristics that speak for a well-differentiated liposarcoma are a size of more than $10 \mathrm{~cm}$, septa less than $2 \mathrm{~mm}$, the presence of nodular, non-adipose solid parts and a total fat percentage of the lesion of less than $75 \%$ [4]. In particular for cases of a recurrent tumor, a tumor size of more than $15 \mathrm{~cm}$ or in an intra-abdominal or retroperitoneal position, a liposarcoma can be differentiated from a lipoma by molecular pathological detection 

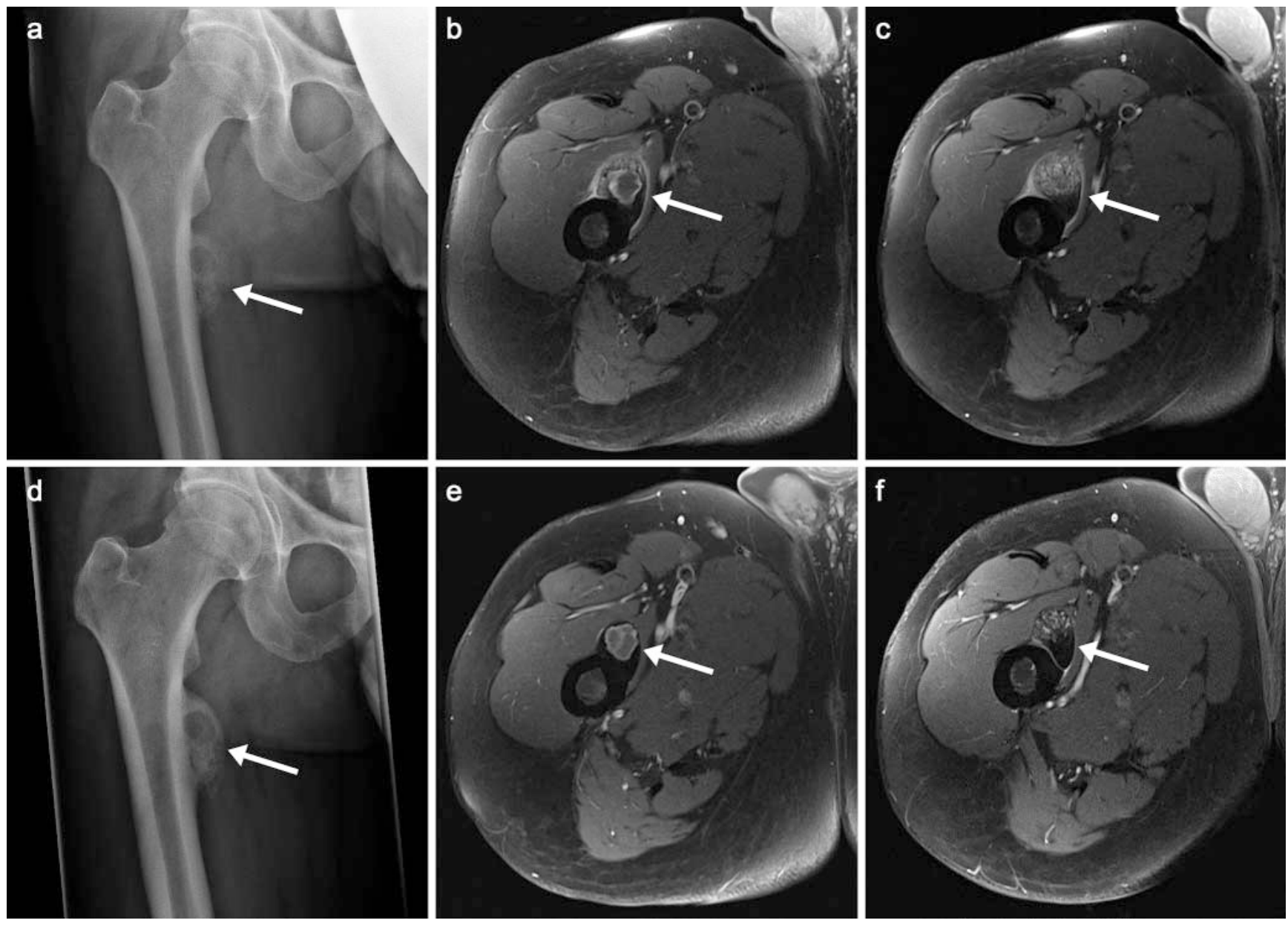

- Fig. 3 47-year-old male with myositis ossificans on right proximal thigh. a In conventional image, juxtacortical, flat calcified formation with cystic whitening cranially and solid portions caudally without cortical erosion (arrow). b, c In contrast-enhanced fat-saturated T1 TSE cranial cystic parts with surrounding enhancement (b, arrow), caudal contrast-enhancing solid elements (c, arrow). d Within 3 months increasing sclerosis (arrow). e, $\mathbf{f}$ In contrast-enhanced fat-saturated axial T1 TSE after 3 months increasing sclerotic zone around the cystic components (e, arrow) with decreasing enhancement and size regression of the solid components (f, arrow).

of MDM2 or CDK4 amplification using fluorescence in situ hybridization (FISH) [8].

\section{Fibroblastic/myofibroblastic tumors}

Nodular fasciitis, proliferative fasciitis, proliferative myositis, and myositis ossificans are benign solid fibrous changes, all of which are classified as variants of nodular fasciitis [9], although myositis ossificans is no longer classified as a chondro-osseous lesion [10]. Myositis ossificans occurs more commonly in the large muscles of the lower extremity, and is more likely in young adults. Often no trauma can be found, contrary to the assumptions based on the patient's history [11]. In the course of time, myositis ossificans presents differently in imaging depending on the stage of development. In the first few weeks, calcifications can rarely be distinguished in conventional $\mathrm{X}$-ray or $\mathrm{CT}$. Two to three months later cloudy-irregular densifications develop, which progress from peripheral to a central compression in a so-called "zonal pattern" and finally can be delimited centrally as an osteoid matrix, as a periph- eral rim of lamellar bone $[12,13]$. The MRI image changes analogously to the described histological changes. In their early stages, the lesions are blurred, isointense on the $\mathrm{T} 1 \mathrm{~W}$, and heterogeneously hyperintense on the $\mathrm{T} 2 \mathrm{~W}$ with diffuse surrounding soft tissue edema. As the calcifications in the periphery increase over time, the signal reduction becomes increasingly apparent. Both the T1w and T2w images show "mature" myositis ossificans as a well-circumscribed soft tissue mass, centrally fat-isointense and with low signal intensity in the periphery without surrounding edema. If central signal levels are also low, fibrosis, mineralization or hemosiderin may be the cause. - Fig. 3 presents the typical progression of the disease. Early forms of myositis ossificans can absorb contrast agent and be misinterpreted as malignant sarcoma [13]. This early stage often presents problems for the histopathologist, especially if initially the clinical picture suggests a progressive tumor and the diagnosis must be made only using a small biopsy [14]. 


\section{Vascular tumors}

Hemangiomas are the most common benign tumors among newborns and infants, with a frequency of $10 \%$ in full-term infants and up to $30 \%$ in preterm infants. Females are three times more likely to be affected. Sixty percent of all hemangiomas occur in the head and neck area, $25 \%$ in the extremities and about $15 \%$ in the trunk; the exact mechanism of origin is not yet sufficiently understood [15].

The concept "hemangioma" thus characterizes various vascular anomalies. The ISSVA classification [16] published in 2014 and revised in May 2018 combines both the classification of congenital vascular tumors and vascular malformations that together make up the group of congenital vascular anomalies. Thus, for example, infantile hemangiomas are classified according to stage, whereas vascular malformations are classified as capillary, venous, lymphatic, arterial or combined malformation of the vascular system according to their growth behavior, localization or organ affiliation as well as the predominant vessel origin [17]. The ISSVA classification focuses on the genetic causes as well as microscopic and radiological findings, and additionally includes currently known genetic disorders. It has been shown that the majority of genetic vascular malformations have disorders in the Ras signaling pathway, similar to the neuroectodermal syndromes, resulting in common therapeutic concepts [17].

Clinically hemangiomas can appear as bluish skin changes and change size over time. Sometimes they can also be painful. In imaging, hemangiomas can contain tortuous vessels, fat, smooth muscle, hemosiderin and phleboliths [18]. Phleboliths disclosed in a conventional X-ray or $\mathrm{CT}$, corresponding to mineralized thrombi, can assist in determining a diagnosis [19]. Bone changes such as periosteal reactions, cortical and medullary changes as well as increased bone growth may be visible [18]. In MRI, hemangiomas typically have a lobular structure in T2w which is considered non-specific; punctate or reticular signal loss can exhibit portions with fibrosis, rapid flow within the vessels or calcification ( $\triangleright$ Fig. 4).

Fluid levels can be signs of cavernous hemangiomas [19]. In T1w images hemangiomas are usually sharply demarcated or with blurred margins; the signal intensity in T1w varies depending on (reactive) fat increase or hemorrhage within the hemangioma [20]. Low-flow hemangiomia components typically appear strongly hyperintense in $\mathrm{T} 2 \mathrm{~W}$ versus fast flow portions that show signal loss [21]. MRI can divide vascular malformations into lowflow and high-flow lesions; in this case dynamic contrast-based MR angiography is used [19]. However, other vascular tumors such as Kaposi's sarcoma and epithelioid hemangioendothelioma have no distinct appearance on MRI and may be deceptively similar to a solitary hemangioma [19].

\section{Fibrohistiocytic tumors}

As the name suggests, this type of tumor occurs on the tendon sheaths, and is a benign proliferative synovial syndrome of joints, bursa and tendon sheaths. It takes a focal shape which is also known as a giant cell tumor of the tendon sheath, distinguished

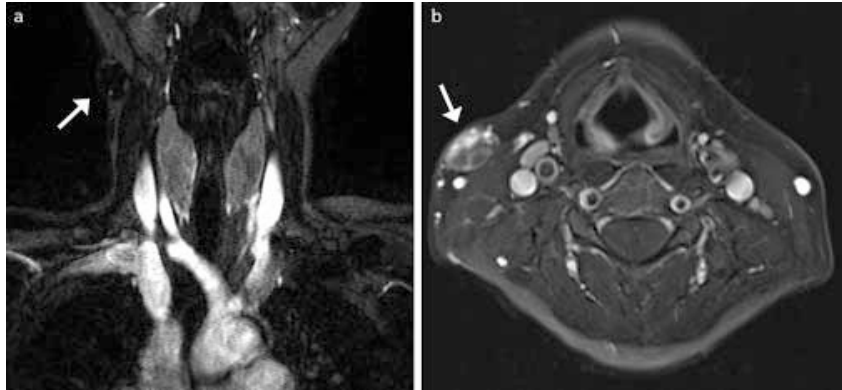

- Fig. 4 35-year-old female with subcutaneous cervical hemangioma. a In coronal early arterial T1 FLASH 3 D angiography sequences evidence of punctate hyperintensities corresponding to feeding vessels (arrow). b The contrast-enhanced fat-saturated T1 axial spin echo shows an inhomogeneous, sometimes very strong enhancement of the lobulated lesion (arrow).

from a diffuse form, pigmented villonodular synovitis. A monoarticular lesion is found in $80 \%$ of all patients [4]. This lesion usually occurs as slowly-growing soft tissue with or without pain. In an $X$-ray image, the localized intra-articular form is unremarkable in most cases [22]. Although the diffuse form shows no abnormalities in conventional images in $21 \%$ of cases [23], in the majority, however, the radiograph reveals joint effusion, periarticular soft tissue swelling, or remodeling of the bone structures adjacent to the joint [24-27]. Further diagnostic imaging using MRI shows typical changes in the form of heterogeneous, diffuse, often nodular synovial thickening typically due to collagen accumulation and hemosiderin inclusions, intermediate to low signal intensity on T1-weighted images and hypointense on T2-weighted images [28]. This effect is particularly evident in gradient echo sequences due to so-called artifact-induced blooming [29]. However, the iron content of the lesions is variable, whereby the imaging characteristics are more or less pronounced. However, when detected, they are regarded as almost pathognomonic for PVNS, since the few other differential diagnoses such as synovial hemangioma additionally have tubular-tortuous vascular structures, and hemophilic arthropathy can be easily excluded by a specific history $[18,29]$.

\section{Peripheral nerve sheath tumors}

Peripheral nerve sheath tumors (PNST) can be benign or malignant. Common to all nerve sheath tumors is the fact that, for a corresponding diagnosis, the close positional relationship to the nerve path must be present and also proven. If this is not the case, the tumor entity plays only a minor role in the differential diagnostic considerations. The benign variants neurofibroma and schwannoma together make up $10 \%$ of benign soft tissue tumors [30]. Schwannomas and neurofibromas can be difficult to differentiate in imaging, as both appear as a circumscribed spindleshaped soft tissue proliferation along a nerve. In some cases, the spatial relationship of the PNST to the nerve may also help in further subdifferentiation. A schwannoma is located rather eccentric to the nerve, a neurofibroma is localized within the nerve [31] ( Fig. 5). 

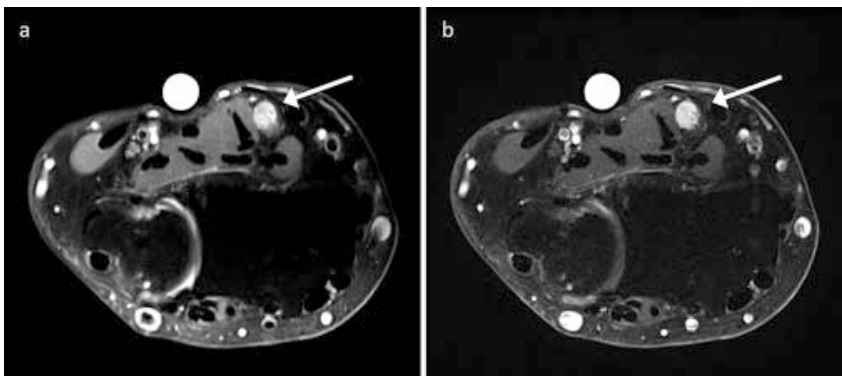

- Fig. 5 49-year-old female with schwannoma eccentric to the median nerve. a In axial PD TSE hyperintense ovular soft tissue proliferation (arrow). b In axial T1 TSE after administration of contrast medium, a strong homogeneous enhancement (arrow) is shown.

Worth mentioning is the so-called "split fat sign", which can be associated with a PNST. As the tumor grows in size, a surrounding marginal wall of normal adipose tissue remains [19]. Benign peripheral nerve sheath tumors are typically isointense to the muscle in T1w images and slightly hyperintense to adipose tissue in T2w [19]. Some benign PNSTs, especially neurofibromas, exhibit a so-called "target sign" in T2w. The T2 W hypointensities are based on the presence of fibrocollagen fibers, while T2w hyperintensities in the margins reflect myxomatous tissue. Benign PNSTs can absorb contrast media [32]. Malignant PNSTs make up at least $6 \%$ of all soft tissue sarcomas [32], and in $50 \%$ of cases associated with type 1 neurofibromatosis [31]. Malignant PNSTs can sometimes be difficult to distinguish from the benign variant. Indicative of a malignant PNST are large lesions with overall rapid growth in size, a blurred border and central necrosis [19, 31].

\section{Magnetic resonance imaging and sequencing protocol}

Good advance planning is recommended prior to selecting the sequences to be used. A palpable mass should be marked with a capsule. If a mass is primarily to be detected, a larger FOV (field of view) should initially be selected and the contralateral side should likewise be imaged. Subsequently, it is necessary to better characterize the lesion using a smaller FOV and higher spatial resolution.

Images in the transverse slice illustrate the anatomical conditions within the examined compartment and evaluate possible environmental infiltration. Coronal or sagittal slices place the mass in relation to anatomical landmarks such as bones, vessels or nerve structures.

As with most other musculoskeletal issues, T1- and T2-weighted images as well as fat-suppressed sequences are required to clarify a soft tissue tumor. In the majority of cases, soft tissue tumors show a hypo- or isointense signal in T1-weighted and a hyperintense signal in T2-weighted images. In the T2-weighted spin-echo or turbo-spin echo sequences, a higher contrast between lesion and surrounding adipose tissue can be achieved by the use of fat-suppressed techniques. An exception to this typical signal behavior for all tumors are those with a high fat content (high signal intensity in the T1-weighted image) or bleeding tumors (high signal intensity of methemoglobin in the T1-weight- ed image, low signal intensity of deoxyhemoglobin and hemosiderin in the T2-weighted image). ${ }^{*}{ }^{*}$ weighted gradient echo sequences can be used to assess hemosiderin deposits found typically in tenosynovial giant cell tumors or, as mentioned above, also in hemangiomas.

The use of gadolinium-based contrast agents helps to better characterize the tumor (see below). In general, MRI should be performed prior to biopsy, as edema and bleeding after biopsy, as well as changes in the biopsy channel may lead to diagnostic problems. The sequence protocol we use is shown in $>$ Table 1.

\section{MRI-based characterization of an indistinct soft tissue tumor}

\section{T1 hypointense or isointense}

Since most soft tissue tumors are iso- or hypointense compared to muscle structures, accordingly the number of potential differential diagnoses is large, ranging from benign lesions such as a ganglion to malignant conditions such as fibrosarcoma ( $\triangleright$ Fig. 6).

Therefore a definite characterization based on a low SI in T1w alone is therefore not possible [33]. The next step involves further clarification by means of T2-weighted sequences.

\section{T1 hyperintense}

In general, signal intensities should be determined on the non-fatsaturated MRI images, as some masses in sequences without fat saturation are isointense to the muscle, but on fat-saturated T1w images they are relatively hyperintense to the muscle. Elements that cause T1 signal attenuation are fat, methemoglobin, protein-rich fluids and melanin. Consequently, differential diagnoses may include fat-containing masses, hemorrhagic masses with methemoglobin components, protein-rich fluids and melanomas or melanoma metastases $[3,11,34]$. In a next step, it should be investigated whether this T1 signal increase can be suppressed by fat saturation. If this is the case, the lesion contains fat, and the most likely diagnoses are lipoma or lipoma variants, a well-differentiated liposarcoma or hemangioma.

Important here is frequency-selective fat saturation (chemically specific), since inversion recovery fat suppression is unspecific and not only fat, but also other substances with short T1 time are suppressed. The diagnosis of a lipoma may be made if the lesion consists largely of fat, contains no or only a few thin septa and no solid, nodular parts. However, if the lesion has a diameter of more than $10 \mathrm{~cm}$, has septa of more than $2 \mathrm{~mm}$ thickness or nodular, non-fatty elements and consists of less than $75 \%$ total fat, the diagnosis of a well-differentiated liposarcoma is likely [4] ( Fig. 7).

\section{T2 hypointense}

A mass that exhibits lower signal intensity compared to skeletal muscle in T2-weighted sequences may include fibrosis, hemosiderin and calcifications. Air and certain foreign bodies are hypointense in the $\mathrm{T} 2 \mathrm{w}$ images. A variety of benign and malignant lesions may exhibit fibrosis, including scar tissue, fibroids and some fibrosarcomas. 
- Table 13 Tesla MRI protocol used in-house for unclear soft tissue tumor of the ankle joint.

\begin{tabular}{|c|c|c|c|c|c|}
\hline sequence & $\mathrm{TR}$ (ms) & TE (ms) & ETL (ms) & $\begin{array}{l}\text { flip angle } \\
\text { (degree) }\end{array}$ & matrix \\
\hline $\begin{array}{l}\text { sagittal T1w TSE before/after contrast } \\
\text { administration }\end{array}$ & 533 & 14 & 3 & 150 & $150 \times 150$ \\
\hline $\begin{array}{l}\text { axial T1w TSE fatsat after contrast } \\
\text { administration }\end{array}$ & 672 & 12 & 3 & 150 & $169 \times 169$ \\
\hline axial T2w TSE & 5000 & 99 & 21 & 157 & $170 \times 170$ \\
\hline coronal T2w TIRM & 5320 (TI 202) & 42 & 10 & 145 & $135 \times 180$ \\
\hline sagittal PDw TSE fatsat & 3350 & 36 & 12 & 178 & $180 \times 180$ \\
\hline axial EP2D: 0-400-800 & 4100 & 76 & 53 & 90 & $220 \times 220$ \\
\hline
\end{tabular}

TSE = Turbine Spin Echo, PD = Proton Density, $w$ = weighted, fatsat = with fat suppression, TIRM = Turbo Inversion Recovery Magnitude .
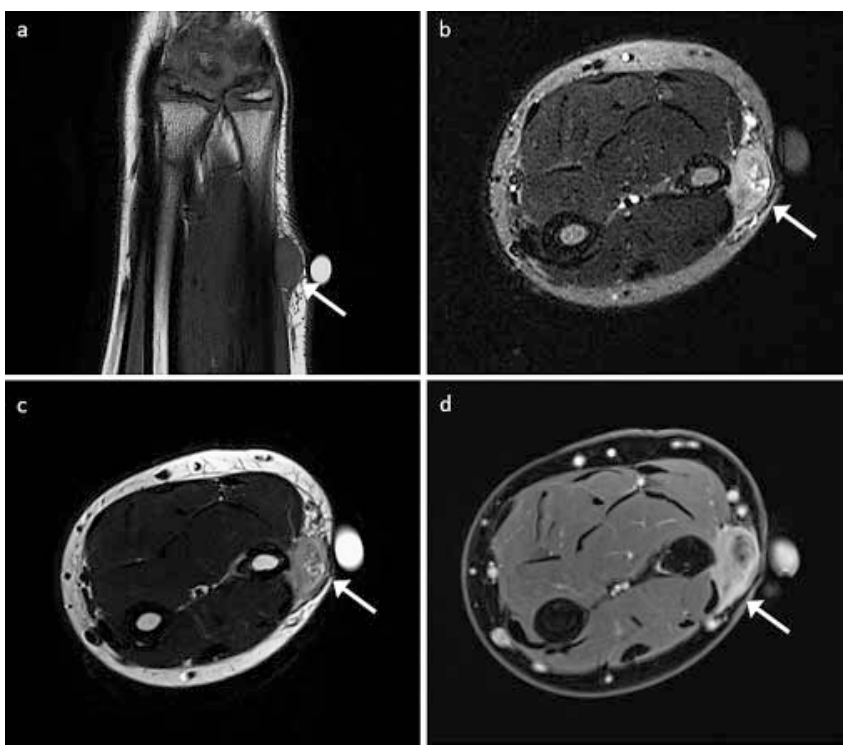

- Fig. 6 7-year-old female with juvenile fibrosarcoma in the subcutis of the forearm. a In axial T1 TSE homogeneous hypointense lesion (arrow). $\mathbf{b}$ In axial TIRM detection of central signal increases (arrow). c In axial T2 TSE moderately hyperintense ovular mass with heterogeneous signal behavior (arrow). $\mathbf{d}$ In axial T1 TSE after contrast administration, strong enhancement of the lesion (arrow) with a central recess, in combination with probable central necrotic melting; perilesional contrast absorption as in the start of ambient infiltration.

Lesions with low Tw2 signal intensity such as a tenosynovial giant cell tumor or lymphoma, are so-called hypocellular fibroses as opposed to hypercellular fibroses, such as in the case of a desmoid, which are hyperintense on T2w images [28, 35-37]. In the case of hemosiderin, $\mathrm{T} 2$ signal reductions can be partially distinguished only in $\mathrm{T} 2 *$ in the form of a "blooming", since the proportion of hemosiderin within the lesion, e. g. in a tenosynovial giant cell tumor or hemangioma may be too low to be detected on T2-weighted images, and $\mathrm{T}^{*}$ is much more sensitive to this. As a rule, calcifications exhibit low T2 signal intensity, signal behavior
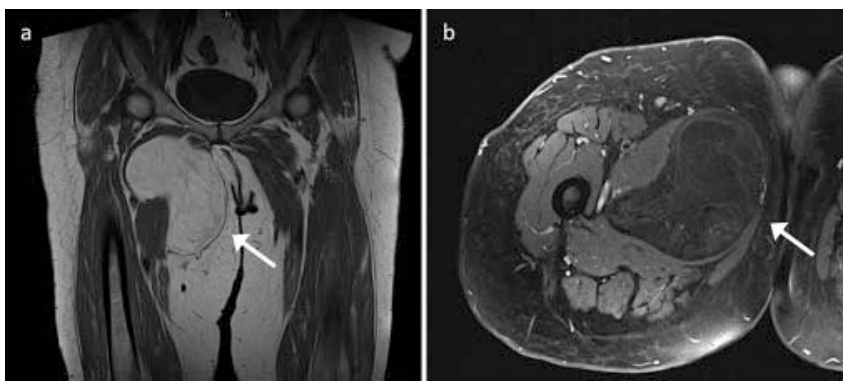

- Fig. 7 68-year-old female with well-differentiated liposarcoma in the adductor compartment. a In coronal T1 TSE, the fat-isoequivalent signal behavior of the smoothly-bounded lesion (arrow). $\mathbf{b}$ In T1 TSE fatsat after administration of contrast medium, signal drop of the fatty mass, enhancement of fine inner septations (arrow).

is dependent on both the extent and distribution of calcification within the lesion, and whether there is an environmental reaction such as edema or an inflammatory response. In their study, Martinez et al. discovered that heterogeneous $\mathrm{T} 2$ signal intensities occur in the context of intratumoral calcifications with hypo- and hyperintense components, whereby the latter can probably be evaluated in the context of inflammatory conditions, such as with foreign body reactions [38]. If a mass exhibits low T2 signal intensity, an X-ray image should be obtained to analyze the presence of calcification, as this is often difficult to do in an MRI. If there is no calcification in the X-ray image, then this is a T2w hypointense lesion, most likely a local fibrosis or a tumor with a substantial component of fibrotic fibers, such as a fibrotic scar after surgery or a tenosynovial giant cell tumor with visible relation to the tendon ( $\vee$ Fig. 8 ).

\section{T2 hyperintense, cyst-like lesions}

Many T2w hyperintense lesions are heterogeneous with diverse differential diagnoses and include liquid lesions such as lymphangiomas, partially liquid lesions such as synovial cysts and seromas, as well as solid lesions such as myxoid sarcoma and synovial sarcoma. Since these solid lesions just mentioned show a rather homo- 

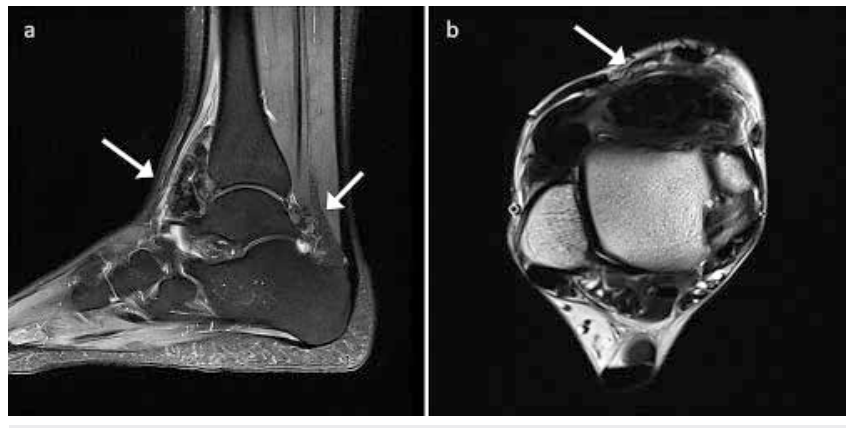

- Fig. 8 24-year-old female with tenosynovial giant cell tumor on the ankle. a In the PD TSE sagittally signal-lowered polylobulatory mass ventrally and dorsally of the tibiotalar joint space (arrow). b Axially severely hypointense nodular soft tissue proliferation in the T2 TSE, consistent with hemosiderin deposits (arrow).

geneous, T2w hyperintense signal behavior, it is important to avoid the misdiagnosis of a fluid-filled structure or a cystic lesion [39]. Hyaline cartilage and hyperemic synovia can likewise imitate fluid $[13,40]$. Intravenous contrast media can distinguish genuine cysts from solid masses [41]. Unlike solid structures, cysts and fluid-filled components do not absorb contrast. However, the MRI acquisition should be performed promptly after administration of contrast agent, since contrast progresses through diffusion into the cyst interior with the passage of time [42]. A classical cyst is a hyperintense T2-weighted lesion with a thin, smooth, contrast-enhancing boundary. If there is a periarticular association, then a ganglion is highly likely, otherwise a differential diagnosis should take into account post-traumatic cysts, seromas, epidermoid inclusion cysts, lymphoceles and lymphangiomas. If the margin is very pronounced with heavy contrast medium absorption and irregular borders, then infectious-inflammatory events such as an inflamed ganglion, an infected hematoma / abscess or tumor necrosis must be considered [43-46] ( $>$ Fig. 9). If not only the margin, but also the entire lesion exhibits strong homo- or heterogeneous enhancement, then a differential diagnosis should consider the possibility of myxoma, myxoid sarcoma, synovial sarcoma or a peripheral nerve sheath tumor [39, 47 -49]. Characteristics of cystic lesions suggestive of malignant disease are a maximum diameter of more than $10 \mathrm{~cm}$ and heterogeneous signal behavior in T1-weighted MRI images [39].

\section{Contrast medium absorption/additional morpho- logical characteristics}

The data show that all larger $(>3 \mathrm{~cm})$ and all subfascial solid soft tissue tumors require MRI with contrast administration, as this provides the highest diagnostic value [50]. The administration of contrast helps to distinguish cystic from solid parts and to detect tumor nodules within cystic lesions. Furthermore, the extent of contrast medium uptake provides information on the degree of vascularization of the lesion and should be mentioned in particular in the preoperative planning. The use of dynamic MRI can especially show the surgeon the location of the vital tumor tissue, and therefore the best site for biopsy. Although malignant tumors tend to show greater enhancement, there is still much controver-
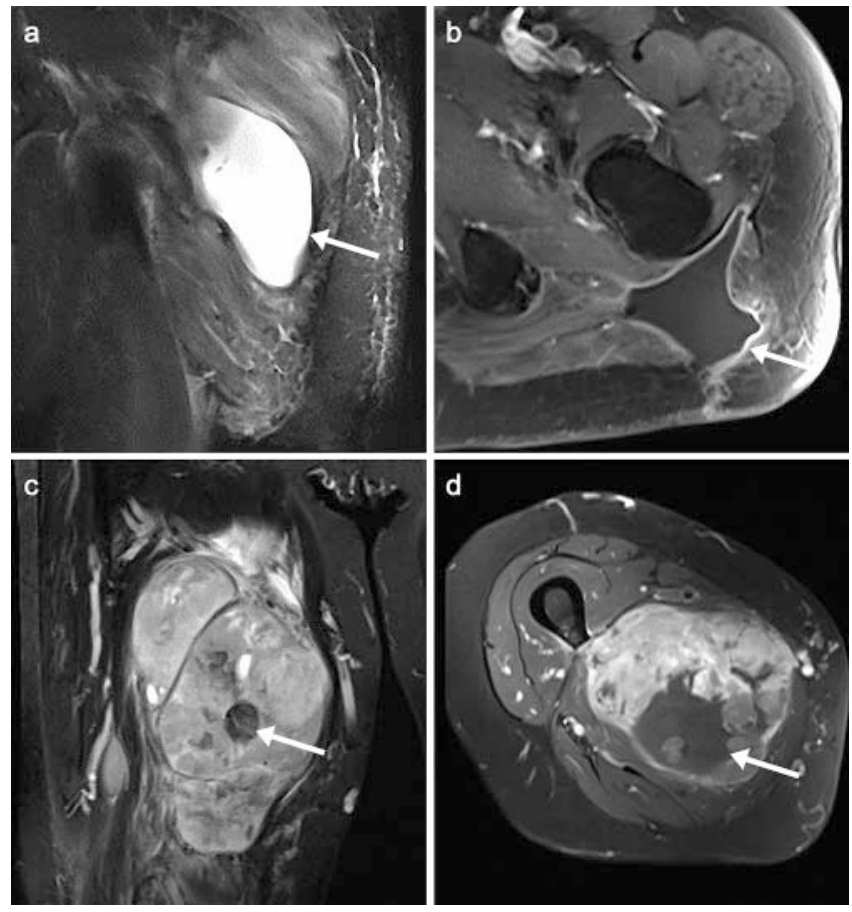

- Fig. 9 Seroma versus tumor necrosis. a, b 63-year-old male with left gluteal resection of a liposarcoma with postoperative seroma. In coronal TIRM, fluid-equivalent ovular lesion (a, arrow). In axial T1 TSE after contrast enhancement of smooth contoured, thin-walled marginal border (b, arrow). c, d 69-year-old female with centrally necrotic fibrosarcoma in the adductor compartment. Inhomogeneous signal behavior with necrosis in the coronal TIRM (c, arrow). In T1 TSE after contrast administration, partly strong enhancement in addition to cystic blending components (d, arrow).

sy in the literature as to how contrast-enhanced MRI can differentiate benign from malignant diseases. Dynamic MRI has shown promising results regarding the differentiation of benign and malignant soft tissue tumors [18, 41, 51, 52]. Recent techniques, such as MR spectroscopy and diffusion-weighted imaging, have shown promising approaches to the evaluation of unclarified soft tissue tumors and the assessment of therapy response, but are not yet part of the clinical routine [53-55]. It would be beneficial to be able to more accurately characterize sarcomas using noninvasive imaging techniques. It is now known that gene expression within a tumor is usually inhomogeneous due to clonal subpopulations. This so-called tumor heterogeneity influences the therapy response and prognosis [56]. The approach of systematically analyzing image data by means of quantifiable image features offers the possibility of personalized medicine with the advantage of a lack of invasiveness without the risk of a biopsy error. The study by Lisson et al. showed, for example, that MRI-assisted $3 \mathrm{D}$ texture analysis can distinguish enchondromas from low-grade chondrosarcomas [57]. However, image-based texture analysis is currently under study and is not yet used in the clinical routine. Thus, there is an additional need for new, functionally-oriented imaging strategies for optimal therapy planning and evaluation of therapy response. 


\section{Summary}

Soft-tissue tumors are frequently encountered by radiologists in everyday clinical practice, and the multiplicity of benign and malignant entities shows the need to develop a systematic diagnostic algorithm. MRI is the central building block of imaging and, through a structured approach, makes it possible to further narrow down possible differential diagnoses. At the least, a separation into benign and unclear lesions seems possible, whereby the number of invasive clarifications by means of biopsy and CT with its radiation load can be significantly reduced. However, this is still unavoidable in cases with residual uncertainty of benignity.

\section{Conflict of Interest}

The authors declare that they have no conflict of interest.

\section{Literatur}

[1] Breitenseher M. Bildgebende Diagnostik und Therapie der Weichteiltumoren: mit pathologischer Klassifikation, Nuklearmedizin, interventioneller Therapie; 21 Tabellen. Thieme. 2008

[2] Bannasch H, Eisenhardt SU, Grosu AL et al. The diagnosis and treatment of soft tissue sarcomas of the limbs. Deutsches Arzteblatt international 2011; 108: $32-38$

[3] Murphey MD, Carroll JF, Flemming D] et al. From the archives of the AFIP: benign musculoskeletal lipomatous lesions. Radiographics 2004; 24: $1433-1466$

[4] Kransdorf M], Bancroft LW, Peterson J] et al. Imaging of fatty tumors: distinction of lipoma and well-differentiated liposarcoma. Radiology 2002; 224: 99-104

[5] Bancroft LW, Kransdorf M], Peterson |] et al. Benign fatty tumors: classification, clinical course, imaging appearance, and treatment. Skeletal radiology 2006; 35: 719-733

[6] Goldblum JR, Weiss SW, Folpe AL. Enzinger and Weiss's Soft Tissue Tumors E-Book; Elsevier Health Sciences; 2013

[7] Jelinek JS, Kransdorf MJ, Shmookler BM et al. Liposarcoma of the extremities: MR and CT findings in the histologic subtypes. Radiology 1993; 186: $455-459$

[8] Zhang H, Erickson-Johnson M, Wang X et al. Molecular testing for lipomatous tumors: critical analysis and test recommendations based on the analysis of 405 extremity-based tumors. Am J Surg Pathol 2010; 34: $1304-1311$

[9] Wang XL, De Schepper AM, Vanhoenacker F et al. Nodular fasciitis: correlation of MRI findings and histopathology. Skeletal radiology 2002; 31 : $155-161$

[10] Fletcher CDM, Unni KK, Mertens F et al. Pathology and Genetics of Tumours of Soft Tissue and Bone. IARC Press. 2002

[11] Crundwell N, O'Donnell P, Saifuddin A. Non-neoplastic conditions presenting as soft-tissue tumours. Clinical radiology 2007; 62: 18-27

[12] Parikh J, Hyare $\mathrm{H}$, Saifuddin A. The imaging features of post-traumatic myositis ossificans, with emphasis on MRI. Clinical radiology 2002; 57: $1058-1066$

[13] Kransdorf M], Meis JM. From the archives of the AFIP. Extraskeletal osseous and cartilaginous tumors of the extremities. Radiographics 1993 13: $853-884$

[14] Jackson DW. Managing Myositis Ossificans in the Young Athlete. Physician and Sportsmedicine 1975; 3: 56-61

[15] Berlien H, Poetke M, Philipp C et al. Phakomatosen. In: Oldhafer M, Hrsg Transitionsmedizin. Stuttgart: Schattauer; 2015: 169-180
[16] International Society for the Study of Vascular Anomalies. ISSVA classification for vascular anomalies (Approved at the 20th ISSVA Workshop, Melbourne, April 2014, last revision May 2018). Available via http:// www.issva.org/UserFiles/file/ISSVA-Classification-2018.pdf. Accessed 7 Jul 2018

[17] Berlien H, Urban P, Poetke M et al. Klassifikation der Vaskulären Malformationen. Phlebologie 2016; 45: 295-303

[18] Kransdorf M], Murphey MD. Imaging of Soft Tissue Tumors; Lippincott Williams \& Wilkins; 2006

[19] Vilanova JC, Barcelo J, Smirniotopoulos JG et al. Hemangioma from head to toe: MR imaging with pathologic correlation. Radiographics 2004; 24 : $367-385$

[20] Murphey MD, Fairbairn KJ, Parman LM et al. From the archives of the AFIP. Musculoskeletal angiomatous lesions: radiologic-pathologic correlation. Radiographics 1995; 15: 893-917

[21] Papp DF, Khanna AJ, McCarthy EF et al. Magnetic resonance imaging of soft-tissue tumors: determinate and indeterminate lesions. The Journal of bone and joint surgery American volume 2007; 89 (Suppl. 3): 103 115

[22] Myers BW, Masi AT. Pigmented villonodular synovitis and tenosynovitis: a clinical epidemiologic study of 166 cases and literature review. Medicine (Baltimore) 1980; 59: 223-238

[23] Dorwart RH, Genant HK, Johnston WH et al. Pigmented villonodular synovitis of synovial joints: clinical, pathologic, and radiologic features. Am J Roentgenol 1984; 143: 877-885

[24] Al-Nakshabandi NA, Ryan AG, Choudur $\mathrm{H}$ et al. Pigmented villonodular synovitis. Clinical radiology 2004; 59: 414-420

[25] Cotten A, Flipo RM, Chastanet P et al. Pigmented villonodular synovitis of the hip: review of radiographic features in 58 patients. Skeletal radiology 1995; 24: 1-6

[26] Llauger J, Palmer J, Roson $\mathrm{N}$ et al. Pigmented villonodular synovitis and giant cell tumors of the tendon sheath: radiologic and pathologic features. Am J Roentgenol 1999; 172: 1087-1091

[27] Karasick D, Karasick S. Giant cell tumor of tendon sheath: spectrum of radiologic findings. Skeletal radiology 1992; 21: 219-224

[28] De Beuckeleer L, De Schepper A, De Belder F et al. Magnetic resonance imaging of localized giant cell tumour of the tendon sheath (MRI of localized GCTTS). Eur Radiol 1997; 7: 198-201

[29] Murphey MD, Rhee JH, Lewis RB et al. Pigmented villonodular synovitis: radiologic-pathologic correlation. Radiographics 2008; 28: 1493-1518

[30] Kransdorf M]. Benign soft-tissue tumors in a large referral population: distribution of specific diagnoses by age, sex, and location. Am J Roentgenol 1995; 164: 395-402

[31] Banks KP. The target sign: extremity. Radiology 2005; 234: 899-900

[32] Murphey MD, Smith WS, Smith SE et al. From the archives of the AFIP. Imaging of musculoskeletal neurogenic tumors: radiologic-pathologic correlation. Radiographics 1999; 19: 1253-1280

[33] Kalayanarooj S. Benign and malignant soft tissue mass: magnetic resonance imaging criteria for discrimination. J Med Assoc Thai 2008; 91: $74-81$

[34] Enochs WS, Hyslop WB, Bennett HF et al. Sources of the increased longitudinal relaxation rates observed in melanotic melanoma. An in vitro study of synthetic melanins. Invest Radiol 1989; 24: 794-804

[35] White LM, Schweitzer ME, Khalili K et al. MR imaging of primary lymphoma of bone: variability of T2-weighted signal intensity. Am J Roentgenol 1998; 170: $1243-1247$

[36] Sundaram M, McGuire MH, Schajowicz F. Soft-tissue masses: histologic basis for decreased signal (short T2) on T2-weighted MR images. Am J Roentgenol 1987; 148: 1247-1250

[37] Dinauer PA, Brixey C], Moncur JT et al. Pathologic and MR imaging features of benign fibrous soft-tissue tumors in adults. Radiographics 2007; 27: $173-187$ 
[38] Martinez S, Vogler JB 3rd, Harrelson JM et al. Imaging of tumoral calcinosis: new observations. Radiology 1990; 174: 215-222

[39] Harish S, Lee JC, Ahmad M et al. Soft tissue masses with "cyst-like" appearance on MR imaging: Distinction of benign and malignant lesions. Eur Radiol 2006; 16: 2652 - 2660

[40] Stiskal MA, Neuhold A, Szolar DH et al. Rheumatoid arthritis of the craniocervical region by MR imaging: detection and characterization. Am J Roentgenol 1995; 165: 585-592

[41] Kransdorf MJ, Murphey MD. Radiologic evaluation of soft-tissue masses: a current perspective. Am J Roentgenol 2000; 175: 575- 587

[42] Ma LD, McCarthy EF, Bluemke DA et al. Differentiation of benign from malignant musculoskeletal lesions using MR imaging: pitfalls in MR evaluation of lesions with a cystic appearance. Am J Roentgenol 1998; 170: $1251-1258$

[43] Frassica FJ, Khanna JA, McCarthy EF. The role of MR imaging in soft tissue tumor evaluation: perspective of the orthopedic oncologist and musculoskeletal pathologist. Magn Reson Imaging Clin N Am 2000; 8: 915 927

[44] Ma LD, Frassica FJ, Scott WW Jr et al. Differentiation of benign and malignant musculoskeletal tumors: potential pitfalls with MR imaging. Radiographics 1995; 15: $349-366$

[45] Sundaram M, Sharafuddin M]. MR imaging of benign soft-tissue masses. Magn Reson Imaging Clin N Am 1995; 3: 609-627

[46] Jelinek J, Kransdorf MJ. MR imaging of soft-tissue masses. Mass-like lesions that simulate neoplasms. Magnetic Resonance Imaging Clinics of North America 1995; 3: 727 - 741

[47] Beggs I. Pictorial review: imaging of peripheral nerve tumours. Clinical radiology 1997; 52: $8-17$

[48] Blacksin MF, Siegel JR, Benevenia J et al. Synovial sarcoma: frequency of nonaggressive MR characteristics. J Comput Assist Tomogr 1997; 21 : $785-789$
[49] Tateishi U, Hasegawa T, Beppu Y et al. Prognostic significance of MRI findings in patients with myxoid-round cell liposarcoma. Am J Roentgenol 2004; 182: 725 - 731

[50] Misra A, Mistry N, Grimer R et al. The management of soft tissue sarcoma. J Plast Reconstr Aesthet Surg 2009; 62: 161 - 174

[51] Verstraete KL, De Deene Y, Roels H et al. Benign and malignant musculoskeletal lesions: dynamic contrast-enhanced MR imaging-parametric "first-pass" images depict tissue vascularization and perfusion. Radiology 1994; 192: $835-843$

[52] van der Woude HJ, Verstraete KL, Hogendoorn PC et al. Musculoskeletal tumors: does fast dynamic contrast-enhanced subtraction MR imaging contribute to the characterization? Radiology 1998; 208: 821 -828

[53] Kettelhack C, Wickede M, Vogl T et al. 31Phosphorus-magnetic resonance spectroscopy to assess histologic tumor response noninvasively after isolated limb perfusion for soft tissue tumors. Cancer 2002; 94 : $1557-1564$

[54] Einarsdottir H, Karlsson M, Wejde ] et al. Diffusion-weighted MRI of soft tissue tumours. Eur Radiol 2004; 14: 959 - 963

[55] Dudeck O, Zeile M, Pink D et al. Diffusion-weighted magnetic resonance imaging allows monitoring of anticancer treatment effects in patients with soft-tissue sarcomas. J Magn Reson Imaging 2008; 27: 1109-1113

[56] Burrell RA, McGranahan N, Bartek ] et al. The causes and consequences of genetic heterogeneity in cancer evolution. Nature 2013; 501: 338 345

[57] Lisson CS, Lisson CG, Flosdorf K et al. Diagnostic value of MRI-based 3D texture analysis for tissue characterisation and discrimination of lowgrade chondrosarcoma from enchondroma: a pilot study. Eur Radiol 2018; $28: 468-477$ 